\title{
Tropical vegetable fats and butters: properties and new alternatives
}

\author{
Joaquín J SALAS \\ Miguel A. BOOTELLO \\ Enrique MARTÍNEZ-FORCE \\ Rafael GARCÉS \\ Instituto de la Grasa, CSIC, Av. Padre García \\ Tejero, 4, 41012, Sevilla, Spain \\ <jjsalas@cica.es>
}

\begin{abstract}
Tropical fats and butters are characterized by their high contents of saturated fatty acids, which confer to them melting points and rheological properties adequate for the production of high valuable food commodities. We can distinguish 3 groups of tropical fats: those having medium chain fatty acids, like coconut or palm kernel oils, those rich in palmitic acid, like palm oil and its fractions, and those rich in stearic acid like cocoa butter. Modern biotechnology has provided with alternatives to these species in engineered common oil crops enriched in saturated fatty acids and processes aimed to enrich common oils in disaturated TAGs by enzymatic transesterification. The present and future of these new sources of saturated fats are discussed in this work.
\end{abstract}

Key words: palm oil, palm kernel, coconut, cocoa butter, oilseed biotechnology

\section{Introduction}

Vegetable fats and oils are extracted from oil seeds and fruits and are the main lipid source in a healthy human diet [1]. These fats and oils consisted of triacylglycerols (TAGs), whose characteristics depend on their fatty acid composition. Oils rich in unsaturated fatty acids are usually liquid at room temperature, whereas the presence of saturated fatty acids increases the melting point of the fat or oil. In this regard, more than $75 \%$ of the world vegetable lipid production consists of liquid oils, which are used for retailing, frying, canning and preparation of emulsions or margarines [2]. Furthermore, solid fats constitute less than $25 \%$ of the world production of vegetable lipids and their applications are eminently industrial because they are the raw material for the production of structured lipids and confectionary fats that are the base for a great variety of food products. The main sources of solid vegetable fats are palm stearins, palm kernel oil, coconut fat, cocoa butter and other butters coming from any other exotic sources like shea, kokum, mango or illipe. A common factor of all these solid fats and butters is that all they are of tropical origin. The cause of this phenomenon is the close relationship between the
TAG synthesis metabolism and the pathways of phospholipids synthesis. Thus, the esterification of the acyl moieties to the glycerol backbone takes place through the Kennedy pathway that involves successive acylation of glycerol-3-P to yield finally a TAG molecule [3]. The main phospholipids present in plant membranes came from ramifications of this pathway, therefore, TAG and phospholipids display similar fatty acid compositions in a given plant organ. On the other hand, biological membranes require a certain degree of fluidity to be functional and this fluidity depends on the degree of saturation of the fatty acids esterified to membrane lipids and temperature [4]. High levels of saturated fatty acids in membrane lipids are incompatible with low temperatures, due to membranes would become brittle causing cell lysis and death. Therefore, the accumulation of saturated fatty acids in TAGs involve high levels of saturated fatty acids in membrane lipids, which only can be supported by permanent high temperatures of tropical climates.

Tropical fats and butters are solid or semisolid at room temperature because they contain high levels of saturated fatty acids in their TAGs. In this regard, they can be classified on the basis of the predominant saturated fatty acid that they contain. Therefore, there are three main groups of tropical vegetable fats: those containing lauric, palmitic or stearic fatty acid. Fats within each group display different properties and origins and are usually fated to different uses.

\section{Lauric fats}

Lauric fats are those accumulating medium and short chain fatty acids (mainly lauric and myristic) as the main fatty acid in their oils. These fats come mainly from palm kernel and coconut, which displays similar fatty acid composition in their reserve TAGs. Thus, these fats contain around 50\% lauric acid, between 15 and 20\% myristic acid, and $8-15 \%$ of saturated shorter chained fatty acids, which are usually more abundant in coconut fat (table 1, [5]). Another characteristic of these fats is the fatty acid distribution within the TAG backbone. Saturated fatty acids are mainly excluded from the sn-2 position of the TAG molecule in plants. This is not the case of lauric fats, which accounts for high levels of lauric acid in that position. Thus, table 1 indicated that the sn-2 position of coconut accumulated important amounts of saturated fatty acids, above all lauric acid, which was

Table 1. Typical fatty acid composition of palm kernel and coconut oils. Distribution of fatty acids between the sn-2 and sn-1, 3 positions of triacylglycerol in the case of coconut oil. Data taken from reference [5].

\begin{tabular}{|lcllllll|}
\hline & \multicolumn{2}{l}{ Fatty acids $(\mathbf{m o l} \%)$} & & & \\
\cline { 2 - 7 } & C6-C10 & $\mathbf{1 2 : 0}$ & $\mathbf{1 4 : 0}$ & $\mathbf{1 6 : 0}$ & $\mathbf{1 8 : 0}$ & $\mathbf{1 8 : 1}$ & $\mathbf{1 8 : 2}$ \\
\hline Palm Kernel & 8 & 48 & 16 & 8 & 2 & 15 & 3 \\
Coconut & 15.6 & 47.9 & 18.2 & 8.7 & 2.5 & 5.7 & 1.4 \\
sn-2 & 4.1 & 78.2 & 10.2 & - & - & 5.9 & 2.0 \\
sn-1+3 & 21.4 & 32.7 & 22.2 & 13.2 & 4.0 & 5.6 & 1.1 \\
\hline
\end{tabular}


present in that position in amounts close to $80 \%$. Other saturated fatty acids were evenly distributed amongst the sn-1 and sn-3 TAG positions.

These TAG compositions confer to lauric fats melting profiles ranging from 10 to $30^{\circ} \mathrm{C}$ that are not enough for the elaboration of confectionary products. Thus, these fats are usually fractionated to yield a stearin with a melting range around $30^{\circ} \mathrm{C}$, which is used in confectiothat is hydrogenated to increase its content of solids [6]. The versatility and the mouthful of lauric fats make them an excellent base for a variety of food products like chocolate substitutes (CBS), filling creams, toffees, caramels, ice creams, milk products, cream substitutes and being a source of saturated fatty acids for the production of structured lipids and margarines [7]. However, on the negative side, there is a clear scientific consensus about the atherogenic effects of laurate and myristate. These fatty acids increase the levels of blood plasma cholesterol and induce arteriosclerosis. Furthermore, these effects on human health are enforced by the TAG structure found in lauric fats, in which the high levels of saturated fatty acids in sn-2 position assure a quick absorption and nary as cocoa butter substitute and an olein

incorporation of these fatty acids into human membrane lipids [8]. In the case of hydrogenated lauric oleins, the atherogenic effects are even enhanced by the presence of trans fatty acids [9], so the use of this kind of fats in confectionary should be restricted as much as possible. Since they are not recommendable as food, the future of lauric fats maybe relies on being a renewable feedstock for soap and oleochemical industries.

\section{Fats rich in palmitic acid}

The main source of vegetable fats rich in palmitic acid is palm oil. Typically, palm oil is a semi solid fat with high content of palmitic acid in a high oleic background, but still containing important amounts of linoleic acid. The content of palmitic acid ranged around $45 \%$ of total composition with levels of oleic acid close to $40 \%$ (table 2). The fatty acids present in this oil are distributed in a broad variety of TAGs, including trisaturated, disaturated and triunsaturated ones (table 2). This confers to palm oil a very broad melting interval ranging from -20 to $50{ }^{\circ} \mathrm{C}$, which makes it impractical

Table 2. Fatty acid and main triacylglycerol composition of palm oil. Data taken from reference [5].

\begin{tabular}{|c|c|c|c|c|c|c|c|c|}
\hline & Fatty & ids (n & & & & & & \\
\hline & $12: 0$ & $14: 0$ & $16: 0$ & 16:1 & $18: 0$ & 18:1 & $18: 2$ & $18: 3$ \\
\hline Palm oil (malaysia) & 0.2 & 1.1 & 44.1 & 0.2 & 4.4 & 39.0 & 10.6 & 0.3 \\
\hline & Triac & lycerc & $\mathrm{mol} \%$ & & & & & \\
\hline TAGs & PPP & POP & POSt & POO & 000 & & & Others \\
\hline & 5.4 & 29.0 & 5.1 & 22.8 & 4.3 & & & 33.4 \\
\hline
\end{tabular}

P: palmitic acid, O: oleic acid, St: stearic acid.

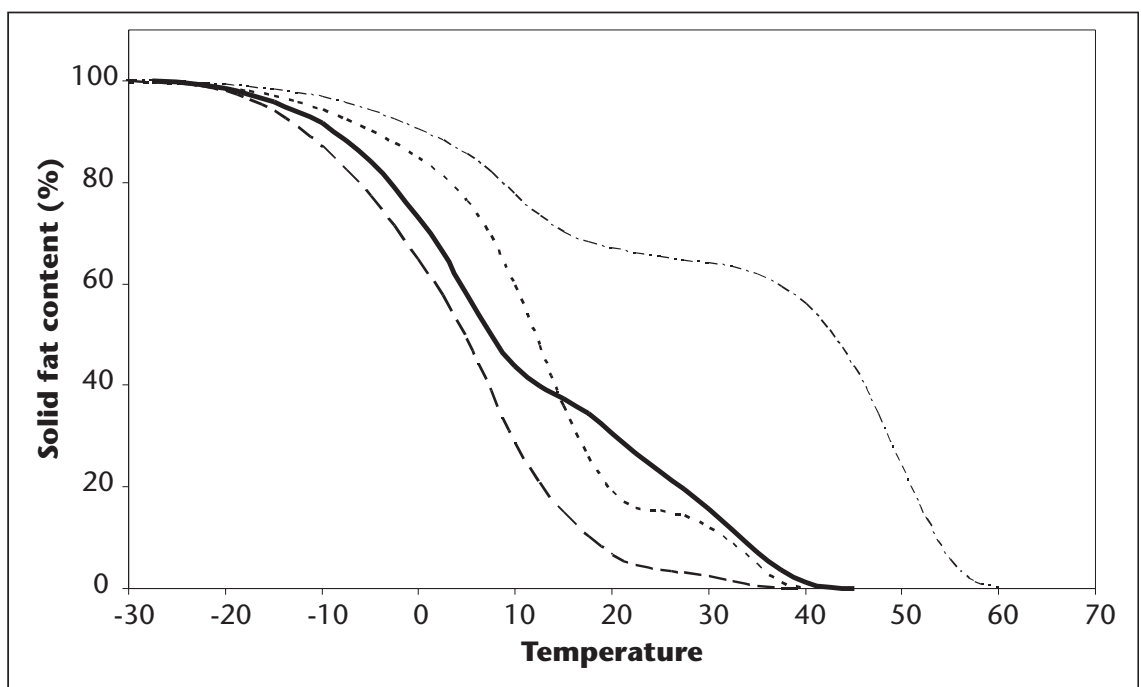

Figure 1. Melting profiles obtained by differential scanning calorimetry of palm oil (solid line), and palm oil fractions. Olein (discontinuous line), palm mid fraction (dotted line) and palm stearin (broken line). for many uses (figure 1). Therefore, palm oil is usually fractionated to obtain fractions with more specific properties [10]. Palm oil fractionation is a complex process, with multiple steps involving the enrichment of certain TAGs on the basis of their different physical properties. Thus, olein liquid fractions are enriched in monosaturated or triunsaturated TAGs and they are liquid or semi liquid at room temperature (figure 1), being usually fated to frying and retailing [11], stearins are enriched in disaturated and trisaturated TAGs and are solid fractions (figure 1) used as source of saturated fatty acids to harden oils in order to produce structured fats [12]. Palm mid fractions (PMF) are enriched in disaturated TAGs, and are extensively used to produce confectionary fats [10]. The composition of palm oil and the versatility of the fractionation process allow producing fats with a variety of characteristics at very competitive prices, which are making palm fractions to displace other oils from international market. In this regard, palm oleins are more stable than most vegetable oils [13]. Palm stearins are widely used to prepare shortenings and margarines. Shortenings are prepared by transesterification of palm and palm kernel stearins with other oils, using often a step of hydrogenation to increase the level of solids of the final fats [12]. Margarines are produced by transesterification of palm stearins with liquid oils. Depending on the final use they require different melting profiles (table, pastry or cake margarines) and, as in the case of shortenings, an extra step of hydrogenation could be necessary to reach the desired melting curve. Palm mid fractions display a steep melting profile (figure 1) that make them useful to produce confectionary fats, being the source of palmitic acid-rich disaturated TAGs in formulations of cocoa butter equivalents (CBEs).

Palmitic acid increases the levels of blood plasma cholesterol in a lower extent than C12-C14 fatty acids [8]. Moreover, little saturated fatty acids are located in the $s n-2$ position of TAGs from palm oil and oleins, which are also rich in oleic acid. Thus, there is evidence showing that these oils are not as atherogenic as previously supposed [14]. But many formulations with palm oil involve concentration of the trisaturated TAGs, chemical randomization, transesterification or hydrogenation, which really increases negative effects on human health of the derivatives of this oil $[15,16]$. The increasing concern of consumers all around the word on the impact of different fats on human health makes that the main challenge of this area of food science is producing healthy fats for food commodities by physical means, suppressing hydrogenation. 


\section{Fats rich in stearic acid}

Fats rich in stearic acid are usually referred as butters and the more important tropical butter is that obtained from seeds of the cocoa (Theobroma cacao) fruit. Cocoa butter (CB) is rich in stearic, oleic and palmitic acids (table 3 ). This fat is quite rich in disaturated TAGs (85-90\%), containing very little saturated fatty acids in the sn-2 position (table 3). Since stearic acid does not exert a negative impact on human health, stearic fats are not generally classified as atherogenic [17]. The most important characteristic of $C B$ is its steep melting curve, which displays a narrow melting interval around $35^{\circ} \mathrm{C}$ that confers chocolate and other confectionary fats their typical characteristics (figure 2, [18]). In this regard, CB quickly melts in mouth releasing flavours and creating a refreshing sensation. This fat is also characterized by a complex polymorphism, with six different crystalline forms that give place to five isomorphs and a high number of possible transitions [19]. Cocoa butter production is hampered by its difficult cultivation, low productivity and pest attacks. These facts, together with an increasing world demand, create market tensions, so it would be necessary to find alternatives. Amongst the classical alternatives to cocoa butter are lauric fats or cocoa butter substitutes,

Table 3. Fatty acid and main triacylglycerol composition of coconut oil from different world locations. Data taken from reference [5].

\begin{tabular}{|c|c|c|c|c|}
\hline & Ghana & Coast & Brazil & Malaysia \\
\hline & Fatty acids (mol\%) & & & \\
\hline Palmitic & 24.8 & 25.4 & 23.7 & 24.8 \\
\hline Stearic & 37.1 & 35.0 & 32.9 & 37.1 \\
\hline Oleic & 33.1 & 34.1 & 37.4 & 33.2 \\
\hline Linoleic & 2.6 & 3.3 & 4.0 & 2.6 \\
\hline \multirow[t]{2}{*}{ Palmitic } & 24.8 & 25.4 & 23.7 & 24.8 \\
\hline & Triacylglycerols (mo & & & \\
\hline Trisaturated & 0.7 & 0.6 & trace & 1.3 \\
\hline Monounsaturated & 84.0 & 82.6 & 71.9 & 87.5 \\
\hline Diunsaturated & 14.0 & 15.5 & 24.1 & 10.9 \\
\hline Polyunsaturated & 1.3 & 1.3 & 4.0 & 0.3 \\
\hline POP & 15.3 & 15.2 & 13.6 & 15.1 \\
\hline POSt & 40.1 & 39.0 & 33.7 & 40.4 \\
\hline StOSt & 27.5 & 27.1 & 23.8 & 31.0 \\
\hline
\end{tabular}

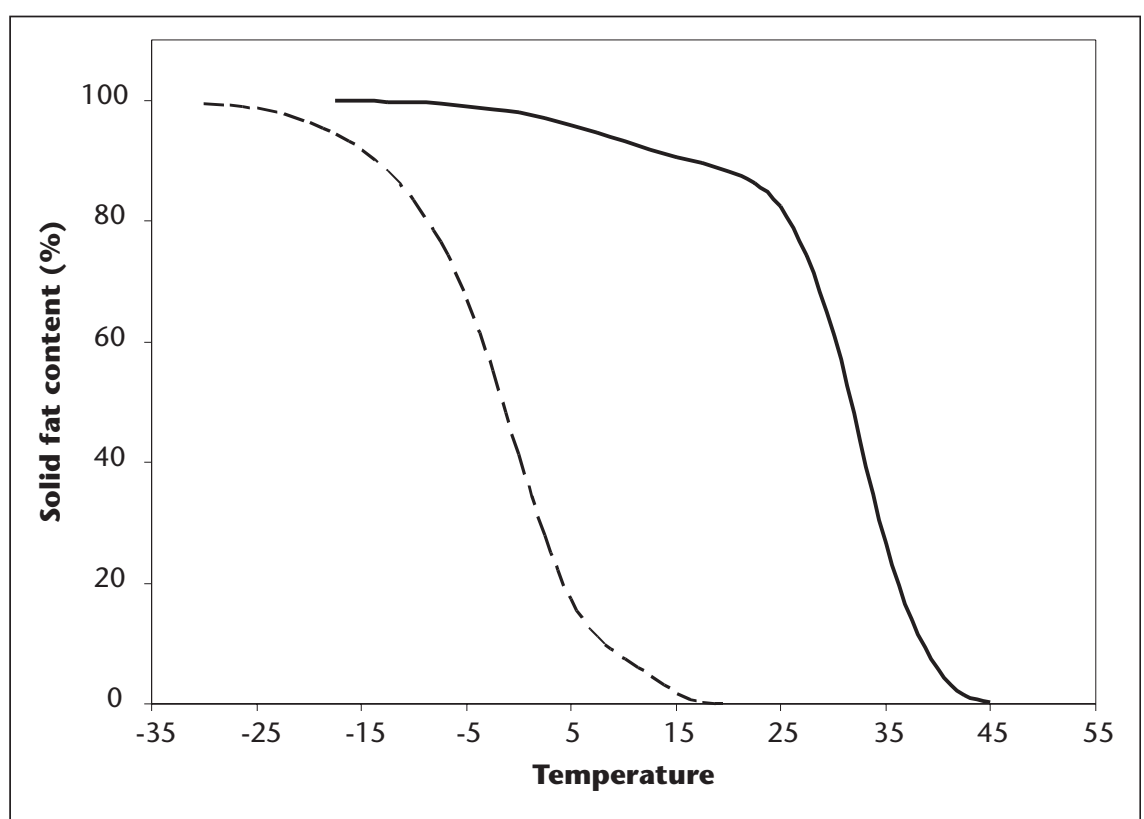

Figure 2. Melting profiles obtained by differential scanning calorimetry of cocoa butter (solid line), and high stearic high oleic sunflower oil (discontinuous line). already mentioned in preceding sections, and hydrogenated oils or cocoa butter replacers (CBR). Lauric CBS increase levels of human blood plasma cholesterol and displays low compatibility when blended with $C B$, giving place to eutectic mixtures. CBRs are more compatible with $C B$, but the tendency of food industry is removing any source of trans-fatty acids in their products. The third alternative are blends of palm mid fractions with fats rich in stearic and oleic fatty acids that can be obtained from certain tropical nuts and seeds like shea, mango, kokum or illipe. These fats are called cocoa butter equivalents (CBEs) and displays similar melting profiles than $\mathrm{CB}$, are fully compatible with it and they do not exert negative effects on human health. Moreover, it is possible to alter the properties of CBEs by manipulating the blending of stearic butters with the added PMFs producing soft and hard CBEs as well as what is called cocoa butter improvers (CBIs), which are butters with a higher melting point that help to keep CB properties in warm climates.

\section{New alternatives to tropical fats}

The uneven distribution of the production of solid fats against liquid oils made that the search for alternatives to tropical solid fats and butters had been an important target of oil industry in the last decades. As it has been depicted above, increasing the melting point of liquid oils by hydrogenation has been the choice of industry for a long time. However, the important concern about the negative effects of trans-fat on human health has made that important health organizations recommended the total removal of these fatty acids from diet [16]. Another alternative to increase the degree of saturation of liquid oils is by the means of transesterification with a saturated fat. This technique is extensively used to produce structured lipids and usually involves the exchange of the acyl moieties by using a chemical catalyser followed of fractionation of the resulting TAGs. This operation usually implies the increment of the saturated fatty acid content in the sn-2 position of the fat product, which in common vegetable oils is usually occupied by an unsaturated one. Increasing the content of saturated fatty acids in the $s n-2$ position increments its absorption in intestine and enables their thorough incorporation into human lipids, negatively affecting cardiovascular health [15]. Nevertheless, new alternatives to tropical fats should be easily accessible, cost effective and compatible with a healthy diet to improve the present status quo. In this regard, advances on biological sciences and 
the understanding of pathways of lipid synthesis has revealed lipid biotechnology as an useful tool to provide with new fats and oils that could compete with those traditionally coming from tropical climates. The pathways and enzymes involved in plant oil synthesis were described trough the 80 's and 90 's decades. Since then, one of the main targets of plant lipid biotechnology has been producing oils enriched in saturated fatty acids in common annual oil crops to cover the growing demand for them [20]. Genetic engineering and mutagenesis techniques allow modifying the TAG composition of oils from many oil crops.

\section{High laurate Canola}

The breakout of biotechnology in the field of tropical fats was quick and very promising. At the beginning of the 90 's Calgene produced lines of canola expressing a thioesterase from Umbellularia californica, which was able to redirect the fatty acid biosynthetic pathway, inducing the accumulation of lauric acid in the oil [21]. Fatty acids are synthesized in the chloroplast or plastids of plant cells in a process involving successive elongations of an acyl-ACP derivative by the action of the fatty acid synthetase. These reactions are terminated by acylACP thioesterases, which are enzymes that hydrolyze acyl-ACP complexes. The fatty acids released are transported to the cytosol in the form of acyl-CoAs and incorporated into TAGs. The expression of a thioesterase from Umbellularia californica in canola seeds ramifies the regular pathway of fatty acid synthesis inducing accumulation of lauric acid in the oil at levels as high as $40 \%$. This oil was commercialized as Laurical and its content of lauric acid and its distribution in the TAG molecule was improved by the introduction of new genes and promoters. This oil was used for the production of soap and surfactants; however, it has not significantly altered the world market of lauric fats, probably because the world demand of these fats is well covered by the current production of coconut and palm kernel.

\section{High palmitic oils}

The great demand of palmitic fats for structured lipid production activated projects for increasing the levels of this fatty acid in common oil crops during the last decade. The enrichment of palmitic acid in non-tropical oil crops is possible by suppressing or reducing $\beta$ ketoacyl-ACP synthase II activity. This is the enzyme catalyzing the last plastidic elongation that takes place in plant fatty acid synthesis, converting 16:0-ACP into 18:0-ACP, which is then desaturated in a great extension by the enzyme stearoyl-ACP desaturase, SAD, and exported to the cell cytosol in the form of oleoyl-CoA. The blockage of this metabolic step by techniques of RNA interaction (hairpin or antisense RNA expression) or gene mutagenesis have been successful at producing oils with increased levels of palmitic acid. These levels ranged 25 to $35 \%$ (table 4 ) in sunflower [22], soybean [23], canola [24] and linseed [25], but none of them reached more than $40 \%$, which would be closer to the composition of palm oil. The cause of this was probably that the enzymes involved TAG synthesis in common oil crops are not able to esterify saturated acyl moieties in the $s n-2$ position of these molecules. On the other hand, soybean, rape or sunflower oils are rich in linoleic acid, therefore it would be necessary to reduce the levels of this fatty acid at expenses of oleic to better mimic the palm oil composition. This can be fulfilled by blocking the oleic acid desaturation. Unlike fatty acid synthesis oleic acid desaturation occurs out of the plastid, through an ER-located desaturase that acts on oleate bound to the $s n-2$ position of phosphatidylcholine, which is called FAD2. The blockage of FAD2 by different means (RNA interaction or mutagenesis) has been achieved in many oil crops, and it could be combined with the high palmitic trait to produce lines such as sunflower CAS-12 combining contents of palmitic acid around $30 \%$ in a high oleic background, containing linoleic acid levels lower than those in palm oil [22]. Although these oils could be a good source of palmitic fats for food industry none of them is currently under exploitation. The reason for this is the great boost of oil palm production that has took place in the Southeast Asia the last years. Thus, palm oil is more abundant and cheap than oil crops growing in temperate climates. Moreover, it displays higher contents of saturates than the above depicted mutants lines, which makes that they did not represent an attractive alternative in this moment.

\section{Oils enriched in stearic acid}

Increasing the content of stearic acid in common oil crops involves the blockage of the step of desaturation of acyl-ACP catalyzed by the enzyme SAD. Equally to the above mentioned oils enriched in palmitic acid, this can be carried out by techniques of genetic engineering (hairpin or antisense RNA technologies) or by mutagenesis of seeds. Decreasing SAD levels during seed development leads to important increases of stearic acid in the seed oil. This has been achieved in several crops like soybean [26], canola [27], cotton seed [28] and sunflower [29]. Moreover, these high stearic phenotypes should be expressed on high oleic backgrounds to mimic better the composition of tropical fats rich in this fatty acid. Therefore, $S A D$ decrease should be combined with the blockage of FAD2 enzyme to yield lines with high stearic-high oleic content in their oils. The stearic acid oil content in the oil of these species was increased from initial 3-6\% to $20-40 \%$ in the mutated or engineered lines (table 5), with contents of oleic acid ranging from 40 to $65 \%$ of total fatty acids. Although the increment in stearic acid reached in these mutant lines was really important, they are not still in the range of the tropical species used as the source of stearic-oleic-stearic (StOSt) TAG for the preparation of confectionary fats, and further increments of total saturated fatty acid content is hampered by the enzymatic machinery in these species, which sometimes is not specific for the accumulation of disaturated TAGs. Furthermore, in the case of "green" seeds, like canola or soybean, a certain content of linolenic acid is always found in the oil

Table 4. Fatty acid composition of different oil crops engineered for the production of palmitic acid.

\begin{tabular}{|lllllllll|}
\hline & \multicolumn{7}{l|}{ Fatty acids (mol\%) } \\
\cline { 2 - 9 } & $\mathbf{1 6 : 0}$ & $\mathbf{1 6 : 1}$ & $\mathbf{1 8 : 0}$ & $\mathbf{1 8 : 1}$ & $\mathbf{1 8 : 2}$ & $\mathbf{1 8 : 3}$ & $\mathbf{2 0 : 0}$ & $\mathbf{2 2 : 0}$ \\
\hline Soybean $[23]$ & 30.4 & - & 5.2 & 10.1 & 42.6 & 11.7 & - & - \\
Canola $[24]$ & 27.0 & 1.2 & 2.8 & 28.8 & 23.2 & 14.0 & 2.0 & 1.0 \\
Sunflower $[22]$ & 30.7 & 7.6 & 2.1 & 56.0 & 3.1 & - & - & - \\
Linseed $[25]$ & 28.4 & 4.0 & 3.8 & 13.5 & 6.3 & 43.0 & - & - \\
\hline
\end{tabular}

Table 5. Fatty acid composition of different oil crops engineered for the production of stearic acid.

\begin{tabular}{|lccclllll|}
\hline & \multicolumn{7}{l}{ Fatty acids (mol\%) } \\
\cline { 2 - 9 } & $\mathbf{1 6 : 0}$ & $\mathbf{1 8 : 0}$ & $\mathbf{1 8 : 1}$ & $\mathbf{1 8 : 2}$ & $\mathbf{1 8 : 3}$ & $\mathbf{2 0 : 0}$ & $\mathbf{2 2 : 0}$ & others \\
\hline Cottonseed [28] & 13.7 & 39.9 & 37.4 & 6.0 & 0.6 & 2.4 & - & - \\
Sunflower [29] & 5.4 & 20.4 & 66.3 & 3.3 & - & 1.7 & 2.8 & - \\
Canola [27] & 7.4 & 19.2 & 62.1 & 4.3 & 1.1 & 3.1 & 0.9 & 1.9 \\
Soybean [26] & 6.0 & 24.0 & 63.0 & 2.0 & 3.0 & - & - & - \\
\hline
\end{tabular}


(1-3\%), this fatty acid is not desirable in confectionary fats because its low melting point and unstability. Other problems reported in oilseed crops enriched in stearic acid referred to seed germination, thus very high levels of this fatty acid have been reported to decrease considerably the rates of germination in antisense mutants from canola plants [27].

High stearic-high oleic oils presents lower saturated fatty acid content than $\mathrm{CB}$, which makes that their melting profiles are far from that found in the butter of that specie (figure 2). However, this have not been an impediment for a growing interest in these oils. Thus, StOSt-rich butters are usually produced from tropical nuts that do not ensure a reliable supply. The levels of saturated fatty acids in the new engineered oils could be increased by techniques of dry or solvent fractionation already applied for other tropical fats like palm, coconut and palm kernel oils. Dry fractionation involves crystallization of the oil at low temperature in absence of any solvent, followed of filtration at high pressure, which allows expelling the liquid olein and enriches the solid fraction in disaturated species of TAGs. In the case of solvent fractionation the oil is dissolved in an organic solvent like acetone or hexane. This micelle is cooled down and the resulting stearin is filtered and washed with fresh solvent. The first type of fractionation is often less efficient but lest costly than the second one. In this regard, high stearic-high oleic sunflower oils, which are being commercialized as healthy stable fats for frying, are especially promising as a starting material for fractionation.

Biotechnology also provides another way to produce StOSt. This is transferring stearic acid to the $s n-1,3$ positions of high oleic oils through enzymatic transesterification. The source of stearic acid would be tallow or fully hydrogenated oils chemically hydrolyzed. These fatty acids can be esterified into the $s n-1$ or $s n-3$ positions of high oleic oils by the action of specific lipase enzymes, giving place to StOSt that could be afterwards purified by fractionation [30]. Lipases from different microorganism are available in market; ie. Lipozyme (Rhizomucor Miehei) and lipases from Aspegillus Niger, Pseudomonas cpacia and Penicillium camembertii. These enzymes can be immobilized for cost optimization and allowed the scaling up of the process for industrial production. This method also suffers of some drawbacks, like cost optimization and European laws, which does not allow the use of the TAGs produced in this way for $\mathrm{CBE}$ production.
Acknoledgements. This work was supported by Advanta Seeds, MICINN and FEDER project AGL 2008-01086/ALI.

\section{REFERENCES}

1. Flickinger $B D$, Huth PJ. Dietary fats and oils: technologies for improving cardiovascular health. Curr Atheroscler Rep 2004 ; 6 : 468-76.

2. Gunstone FD. Vegetable Oils in Food Technology. Boca Raton, Florida, USA : CRC Press, 2002.

3. Ohlrogge J, Browse J. Lipid biosynthesis. Plant Cell $1995 ; 7$ : 957-70.

4. Falcone DL, Ogas JP, Somerville CR. Regulation of membrane fatty acid composition by temperature in mutants of Arabidopsis with alterations in membrane lipid composition. BMC Plant Biol $2004 ; 4: 17$.

5. Gunstone FD, Harwood JL, Dijkstra DJ. The lipid handbook. Boca Raton, Florida, USA : CRC Press, 2007.

6. Siew WL. Crystallisation and melting behaviour of palm kernel oil and related products by differential scanning calorimetry. Eur / Lipid Sci Technol 2001 ; 103 : 729-34.

7. Pantzaris TP, Mohd JA. Properties and utilization of palm kernel oil. Palm Oil Developments $2001 ; 35: 11-23$.

8. Katan MB, Zock PL. Mensink. Dietary oils, serum lipoproteins, and coronary heart disease. Am / Clin Nutr $1995 ; 61$ : 1368S-1373S.

9. De Roos N, Schouten E, Katan M. Consumption of a solid fat rich in lauric acid results in a more favourable serum lipid profile in healthy men and women than consumption of a solid fat rich in trans-fatty acids. J Nutr 2001; 131 : 242-5.

10. Kellens MJ. Oil modification processes. In : Hamm W, Hamilton RJ, eds. Edible oil processing. Sheffield, UK : Academic Press, 2001 : 129-73.

11. Matthäus $B$. Use of palm oil for frying in comparison with other high-stability oils. Eur / Lipid Sci Technol $2007 ; 109$ : 400-9.

12. Aini IN, Miskandar MS. Utilization of palm oil and palm products in shortenings and margarines. Eur / Lipid Sci Technol 2007; 109: 422-32.

13. Ismail R. Palm oil and palm olein frying applications. Asia Pacific / Clin Nutr 2005 ; 14 : 414-9.

14. Ebong PE, Owu DU, Isong EU. Links Influence of palm oil (Elaesis guineensis) on health. Plant Foods Hum Nutr $1999 ; 53$ : 209-22.

15. Bell SJ, Bradley D, Forse RA, Bistrian BR. The new dietary fats in health and disease. / Am Diet Assoc 1997 ; 97 : 280-6.
16. Zaloga GP, Harvey KA, Stillwell W, Siddiqui R. Trans fatty acids and coronary heart disease. Nutr Clin Pract 2006 ; 21 : 505-12.

17. Elson CE. Tropical oils: nutritional and scientific. Crit Rev Food Sci Nutr 1992 ; 31 : 79-102.

18. Shukla VKS. Cocoa butter properties and quality. Lipid Technol 1995May: 54-7.

19. Smith KW. Cocoa butter and cocoa butter equivalents. In: Gunstone FD, ed. Structured and modified lipids. New York: Marcel Dekker, 2001 : 401-22.

20. Drexler H, Spiekermann P, Meyer A, et al. Metabolic engineering of fatty acids for breeding of new oilseed crops: strategies, problems and first results. J Plant Physiol 2003; 160 : 779-802.

21. Voelker TA, Worrell AC, Anderson L, et al. Fatty acid biosynthesis redirected to medium chains in transgenic oilseed plants. Science 1992; $257: 72-4$.

22. Fernández-Martínez JM, Mancha M, Osorio J, Garcés R. Sunflower mutant containing high levels of palmitic acid in high oleic background. Euphytica 1997 ; 97 : 113-6.

23. Fehr WR. Inheritance of elevated palmitic acid content in soybean seed oil. Crop Sci $1991 ; 31$ : 1522-4.

24. Jones A, Davies HM, Voelker TA. Palmitoyl-acyl carrier protein (ACP) thioesterase and the evolutionary origin of plant acyl-ACP thioesterases. Plant Cell $1995 ; 7$ : 359-71.

25. Rowland GG, Bhatty RS. Ethyl methanesulfonate induced fatty acid mutations in flax. JAOCS $1990 ; 67: 213-4$.

26. Knowlton $S$. Fat products from high stearic soybean oil and a method for the production thereof. WO/1999/057990. 1999.

27. Knutzon DS, Thompson GA, Radke SE, Johnson WB, Knauf VC, Kridl JC. Modification of Brassica seed oil by antisense expression of a stearoylacyl carrier protein desaturase gene. Proc Nat Acad Sci USA 1992 ; 89 : 2624-8.

28. Liu Q, Singh SP, Green AG. High-Stearic and High-Oleic Cottonseed Oils Produced by Hairpin RNA-Mediated Post-Transcriptional Gene Silencing. Plant Phys $2002 ; 129$ : 1732-43.

29. Pleite R, Martinez-Force E, Garces R. Increase of the stearic acid content in high-oleic sunflower (Helianthus annuus) seeds. J Agric Food Chem 2006 ; 54 : 9383-8.

30. Seriburi V, Akoh CC. Enzymatic transesterification of triolein and stearic acid and solid fat content of their products. JAOCS $1998 ; 75$ : 511-6. 